\title{
Dynamic control of THz waves through thin-film transistor metamaterials
}

\author{
Fang-Fang Ren*a,b, Wei-Zong Xu ${ }^{\mathrm{a}, \mathrm{b}}$, Hai Lu ${ }^{\mathrm{b}}$, Jiandong Ye ${ }^{\mathrm{a}, \mathrm{b}}$, Hark Hoe Tan ${ }^{\mathrm{a}}$, Chennupati Jagadish ${ }^{\mathrm{a}}$ \\ ${ }^{a}$ Department of Electronic Materials Engineering, Research School of Physics and Engineering, The \\ Australian National University, Canberra, ACT 0200, Australia; ${ }^{\mathrm{J}}$ Jiangsu Provincial Key Laboratory \\ of Advanced Photonic and Electronic Materials, and School of Electronic Science and Engineering, \\ Nanjing University, Nanjing 210093, China
}

\begin{abstract}
We propose a hybrid metamaterial with embedded amorphous oxide thin-film transistor (TFT) arrays, which embraces the advantages of energy saving, low cost and high yields for tunable amplitude modulation in terahertz (THz) regime. The properties of this active metamaterial system are numerically investigated based on full-wave techniques and multipole theory. The calculation results attribute the modulation to a change in the damping rate of an electric dipoletype resonance mode caused by the increased conductivity of the transparent oxide layer. Such a device, expanding the horizon of oxide electronics into metamaterials, opens up many fascinating prospects for producing stable, uniform, and low-cost $\mathrm{THz}$ components.
\end{abstract}

Keywords: THz metamaterials, thin-film transistors, transparent oxides, modulators

\section{INTRODUCTION}

During the last few decades, the terahertz (THz) region of electromagnetic spectrum, lying between $100 \mathrm{GHz}$ and 10 $\mathrm{THz}$, has attracted increasing attention due to its technological potential. Since several solid-state systems have characteristics dynamics belonging to this region, $\mathrm{THz}$ technology is important for spectroscopy, investigation of molecular bonds, and material structures. Efforts are currently underway to meet the requirements of applications, including the development of techniques for higher power $\mathrm{THz}$ generation, more sensitive $\mathrm{THz}$ detection, and more effective control and manipulation of $\mathrm{THz}$ waves ${ }^{1}$. Although, the $\mathrm{THz}$ region is of importance in fundamental scientific research and acting as a bridge between optics and electronics, well-developed technologies at microwave and optical frequencies are incompatible with $\mathrm{THz}$ frequencies.

\subsection{Active THz metamaterials}

In the search of materials for $\mathrm{THz}$ applications, the composite artificial materials termed electromagnetic metamaterials have emerged. In most cases, the essential building block of the metamaterial is a resonant unit cell, and traditional planar metamaterials are often composed of split ring resonators (SRRs), whose resonance can be tuned or controlled by external stimulus like light, bias voltage, temperature, or magnetic field ${ }^{2}$. Given that such schemes of coupled SRRs tend to affect their spectral response, dynamical tuning of metamaterial resonances provides an efficient way to manipulate electromagnetic waves. This is of particular importance for the $\mathrm{THz}$ regime which still suffers from the shortage of devices required to fully exploit its potential applications. For example, imaging at $\mathrm{THz}$ frequencies is a highly soughtafter goal of researchers, considering the great potential for applications ranging from security screening and skin cancer detection to all weather navigation and biodetection. However, more efficient and precision operation of screening or detection both express requirements on large-area-array devices functioning at $\mathrm{THz}$ regime.

*fangfang.ren@anu.edu.au; phone 612612 51154; fax 612612 50511; physics.anu.edu.au/eme/

SPIE Micro+Nano Materials, Devices, and Systems, edited by Benjamin J. Eggleton, Stefano Palomba Proc. of SPIE Vol. 9668, 966800 - @ 2015 SPIE · CCC code: 0277-786X/15/\$18 · doi: 10.1117/12.2202359 


\subsection{Transparent oxide thin-film transistors}

So far, various ways to accomplish active metamaterial modulators for $\mathrm{THz}$ radiation have been demonstrated $\mathrm{d}^{3-5}$. However, most of them are unsuitable for large area fabrication, and actually pose more stringent requirement on simple growth process. On the other hand, thin-film transistors (TFTs) based on amorphous semiconductor materials are the appropriate choice for large area fabrication with good uniformity, and has already been widely used as driver module at high-resolution, high-switching-speed, and large-size active-matrix liquid crystal display. In recent years, there has been great interest in developing TFTs made of ionic amorphous oxide semiconductors, especially the ternary oxide system InGaZnO (IGZO). They have promising electrical performance for TFT active layer with high field effect mobility, low off state current, high on-to-off ratio, and good uniformity compatible with the state-of-the-art Gen- 8 substrate size.

In this work, we propose an active metamaterial capable of efficient real-time control and manipulation of $\mathrm{THz}$ radiation. The device utilizes monolithic integration of TFT at the metamaterial unit cell level and is able to act as an intensity modulator in THz regime. The properties of the hybrid metamaterial system are theoretically investigated based on fullwave techniques and multipole theory. Our results present a new platform for exploring stable, uniform and low-cost modulators in $\mathrm{THz}$ and other frequency range.

\section{DEVICE DESIGN}

\subsection{Schematic of the device}

Figures 1 presents a schematic illustration of the metamaterial unit cell, which consists of two metal layers, separated by a thin dielectric spacer. The top metal layer (see Figure 1(a)) is patterned to form a two-dimensional array of electric resonators and the metal wires in the second layer are uniformly spaced along the $x$ direction (see Figure 1(b)). The metamaterial elements are electrically connected using conducting wires so that the entire metamaterial array functions as drain/source/gate electrodes, separately. The schematic cross section of a unit cell is depicted in Figure 1(c). The thickness of AOS active layer and $\mathrm{SiO}_{2}$ insulating layer is $550 \mathrm{~nm}$ in total. It was found in experiment that a thick $\mathrm{SiO}_{2}$ gate insulator can effectively minimize the implication of back gate metal electrode on the resonant properties of the final metamaterial device.
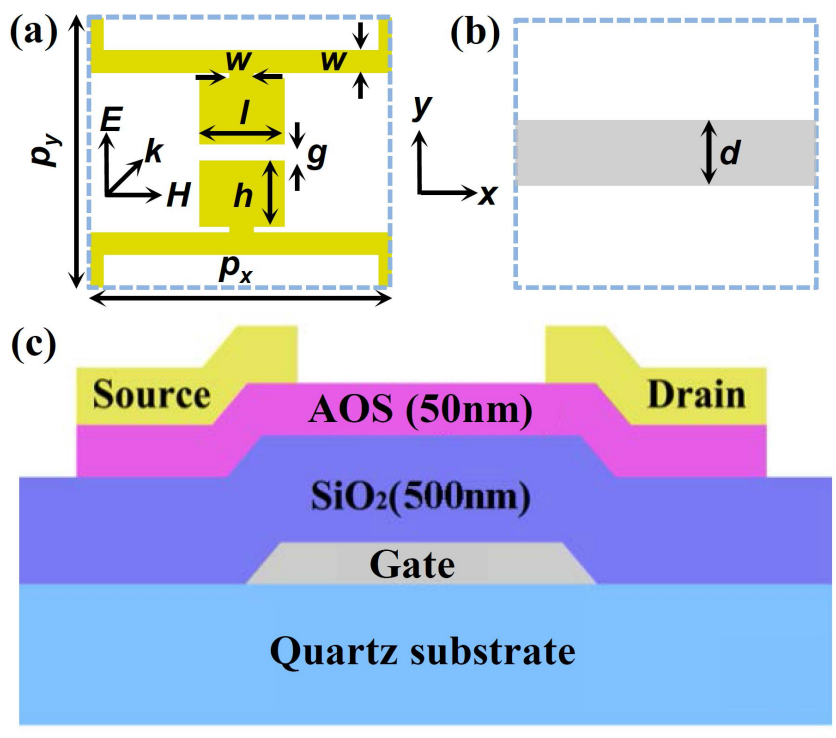

Figure 1. Schematic of (a) the top metal layer and (b) the second metal layer in a metamaterial unit cell. (c) Schematic cross section of a TFT in a unit cell. 


\subsection{Transmission and reflection spectra of the metamaterial without an applied bias}

The metamaterial geometry is optimized as follows: the period $p_{\mathrm{x}}=p_{\mathrm{y}}=50 \mu \mathrm{m}$, the line width $w=4 \mu \mathrm{m}$, the dielectric gap $g=3 \mu \mathrm{m}$, the capacitor dimensions $h=12.5 \mu \mathrm{m}, l=14 \mu \mathrm{m}$, and the gate width $d=11 \mu \mathrm{m}$. For the polarization of incident $\mathrm{THz}$ electric field perpendicular to the split gap, an intrinsic resonance mode at $0.75 \mathrm{THz}$ can be observed in the transmission and reflection spectra as shown in Figure 2. The system exhibits a highly-damped response, which is fairly broadband in nature. To verify the nature of the resonance, we calculated the macroscopic electric and magnetic polarization as function of frequency based on multipole theory. The results identify that the resonance mode at 0.75 $\mathrm{THz}$ in this hybrid structure has an almost purely electric-dipole type response.

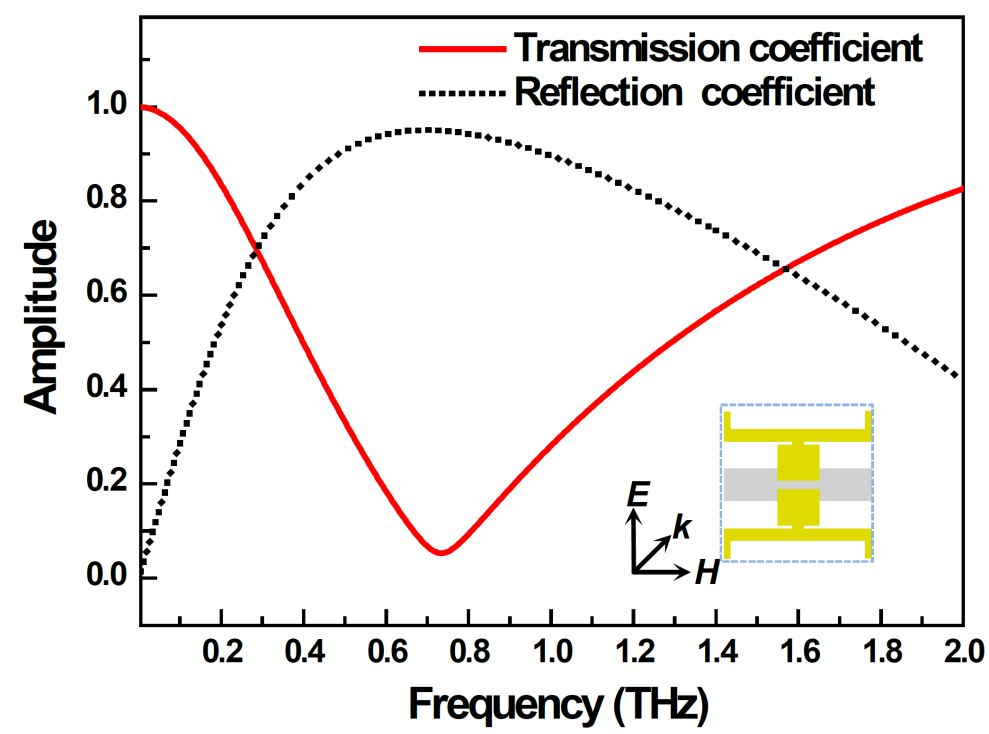

Figure 2. Numerical simulated transmission/reflection coefficient of the TFT-based metamaterials with a completely depleted channel.

\section{ELECTRICAL CONTROL OF METAMATERIALS}

When a positive gate bias is applied to the transistor arrays, the channel currents will flow into the active layer, which has a tunable conductivity. In such a way, the THz electric near-field can be enhanced in the gap of the capacitors and the electromagnetic properties of the resonators can be dynamically tailored.

\subsection{Relationship between the bias and the conductivity}

Figure 3 shows how the conductivity of the IGZO channel increases as the gate voltage sweeps from off-state to on-state with an on-to-off ratio of approximately $10^{7}$. Such a result can be extracted from our previous work ${ }^{6}$. It reveals the modulating ability of gate bias on the conductivity of IGZO and thereby the resonant properties of metamaterials. As the bias is varied from 0 to $24 \mathrm{~V}$, we find the conductivity of IGZO is increased from $4 \times 10^{-4}$ to $40 \mathrm{~S} \mathrm{~m}^{-1}$. For very high conductivities, an alternative channel material such as InZnO (IZO) can be employed in the transistor to form the metamaterial unit, as the conductivity can be tuned up to $5 \times 10^{4} \mathrm{~S} \mathrm{~m}^{-1}$.

\subsection{Modulation performance}

To approximate the implications on the resonance from modification of IGZO channel, we employed the commercially available CST Microwave Studio software to numerically simulate the transmission spectra of the hybrid $\mathrm{THz}$ metamaterial with increasing conductivity in the oxide layer as shown in Figure 4. Here, the differential transmission is defined as $D=\left(T-T_{0}\right) / T_{0} . T$ is the transmitivity, and $T_{0}$ is corresponding to the transmitivity at zero gate bias. In the 
calculation, the IGZO is simulated with the permittivity infinity of $\sim 4$ and the quartz substrate was treated as a loss free dielectric. At zero gate bias, the metamaterial shows significant resonance near the frequency of $0.75 \mathrm{THz}$ (see Figure 2). By sweeping the gate bias from 0 to $24 \mathrm{~V}$ in simulation, we find an enhanced response as the conductivity $(\sigma)$ in the oxide layer is increased. Such results indicate that an increasing forward gate bias will gradually short the capacitive split gap and thereby the electric resonance begins to diminish. At a forward gate bias of $24 \mathrm{~V}$, the differential transmission of 1.75 is obtained, which is 3 orders of magnitude higher than the value at $V$ gate $=4 \mathrm{~V}$. Furthermore, only a small frequency red-shift of about $15 \mathrm{GHz}$ are observed at resonant frequency, making this device a reasonably efficient $\mathrm{THz}$ modulator in this frequency regime.

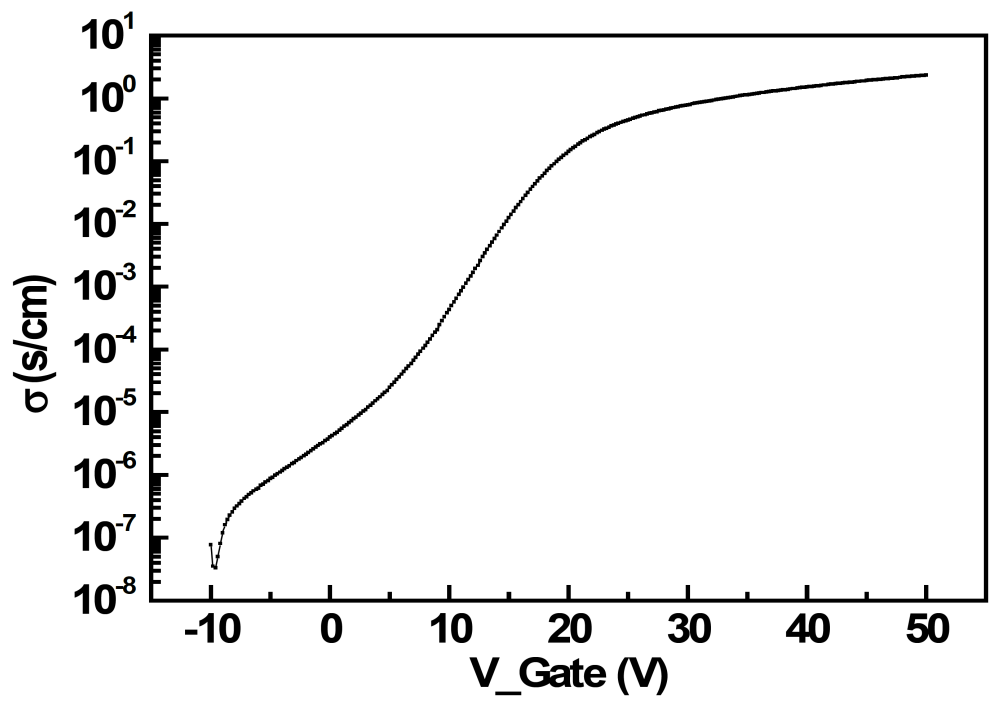

Figure 3 . The conductivity of the IGZO channel with various gate voltages.

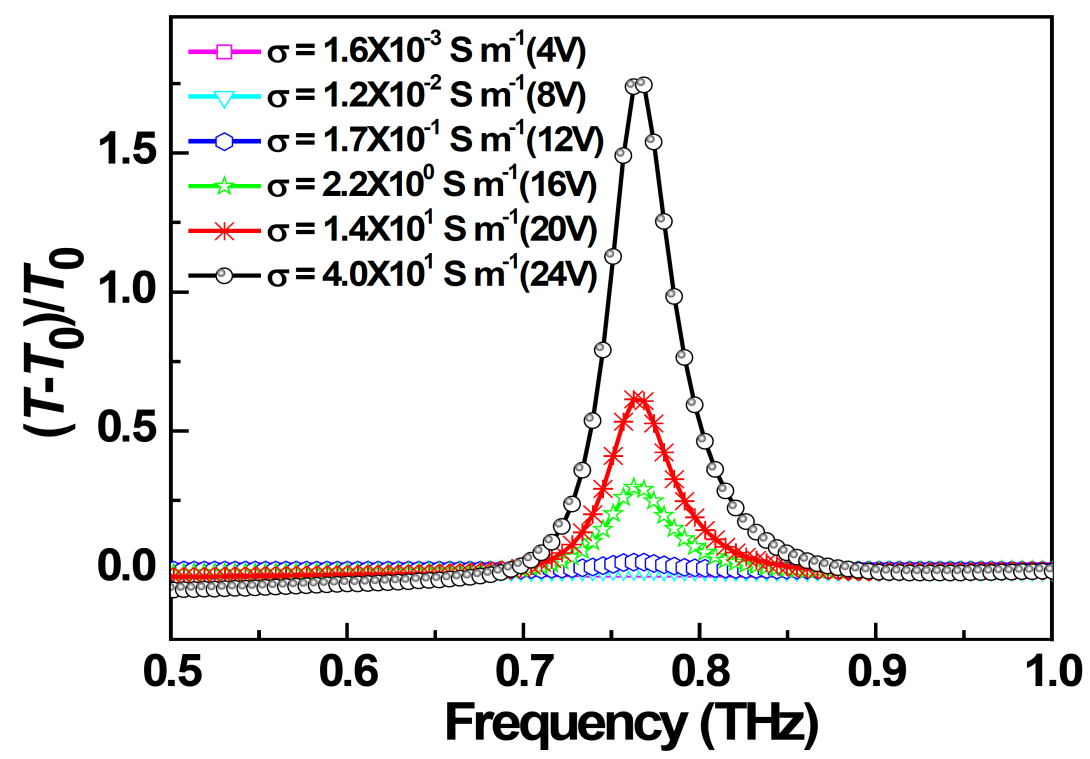

Figure 4. Numerical simulated differential transmission spectra of the hybrid $\mathrm{THz}$ metamaterial with increasing conductivity in the oxide layer. 


\section{REFERENCES}

[1] Chen, H. T., Padilla, W. J., Zide, J. M. O., Gossard, A. C. and Taylor, A. J., "Active terahertz metamaterial devices," Nature 444, 597-600 (2006).

[2] Liu, M., Hwang, H. Y., Tao, H., Strikwerda, A. C., Fan, K., Keiser, G. R., Sternbach, A. J., West, K. G., Kittiwatanakul, S., Lu, J., Wolf, S. A., Omenetto, F. G., Zhang, X., Nelson, K. A. and Averitt, R. D., "Terahertz-field-induced insulator-to-metal transition in vanadium dioxide metamaterial," Nature 487, 345-348 (2012).

[3] Chowdhury, D. R., Singh, R., O'Hara, J. F., Chen, H. T., Taylor, A. J. and Azad, A. K., "Dynamically reconfigurable terahertz metamaterial through photo-doped semiconductor, " Appl. Phys. lett. 99(23), 231101 (2011).

[4] Chen, H. T., Palit, S., Tyler, T., Bingham, C. M., Zide, J. M. O., O’Hara, J. F., Smith, D. R., Gossard, A. C., Averitt, R. D., Padilla, W. J., Jokerst, N. M. and Taylor, A. J., "Hybrid metamaterials enable fast electrical modulation of freely propagating terahertz waves," Appl. Phys. lett. 93(9), 091117 (2008).

[5] Valmorra, F., Scalari, G., Maissen, C., Fu, W., Schönenberger, C., Choi, J. W., Park, H. G., Beck, M. and Faist, J., "Low-bias active control of terahertz waves by coupling large-area CVD graphene to a terahertz metamaterial," Nano Lett. 13, 3193-3198 (2013).

[6] Huang, X., Wu, C., Lu, H., Ren, F., Chen, D., Liu, Y., Yu, G., Zhang, R., Zheng, Y. and Wang, Y., "Largeswing a-IGZO inverter with a depletion load induced by laser annealing," IEEE Elec. Dev. Lett. 35, 1034 (2014). 